\title{
Drive level and cue utilization in spontaneous alternation
}

ROBERT E. FRANKEN AND J, GARRY BAKER UNIVERSITY OF CALGARY
Post-hoc analysis of data from a previously reported experiment suggest that cues utilized in the spontaneous alternation task differ depending on the drive level of the organism. In the previous study drive levels were manipulated to differentially enhance the incentive value of food reward. No effect due to food reward was found irrespective of drive level; however an interaction was found between availability of cues and drive level.

Douglas (1966) has provided data indicating that some of the cues utilized in spontaneous alternation are direction, odor and visual; direction being the most effective, odor of less importance, and visual of little or inconsequential importance. An experiment designed to study the problem of alternation to complex visual stimuli following food rewarded trials (Baker \& Franken, 1967) fortuitously provided data bearing on the problem of drive level and cue utilization. These data may provide an explanation of the failure to obtain the spontaneous alternation effect under certain experimental conditions and differential amounts of exploration of complex stimuli observed under various drive levels.

If direction, odor, and visual cues all govern, in part, the amount of spontaneous alternation, it is necessary to design conditions where the effects of each cue plus the combined effects of cues can be studied separately. The data reported here provide information about three of the possible 8 combinations: (a) all cues available, (b) directional and odor cues available, and (c) visual cues available. For each of the three cue conditions there are three drive levels: (a) $0 \mathrm{~h}$, (b) $4 \mathrm{~h}$, and (c) $22 \mathrm{~h}$ food deprivation. While these conditions are not sufficient to identify the exact relation between drive level and cue utilization, they are sufficient to determine whether there is a relation.

\section{Method}

The Ss were 45 male and 45 female naive blackhooded rats about 90 to 120 days of age and supplied from the National Laboratory Supply in Edmonton, Alberta. The Ss were randomly assigned to one of the nine experimental groups so that each drive-cue condition had five male and five female Ss.

The apparatus was a simple $T$ maze of the following dimensions: height, 8 in.; width, 6 in.; length of stem, 28 in. (the first $8 \mathrm{in}$. of the stem was separated from the rest of the stem by a clear plastic door in order to form a start box); each arm, 18 in. with the last $8 \mathrm{in.}$ of the arm also 8 in. wide so as to form a distinguishable goal box. Clear plastic doors at the union of the arms and the stem were employed to retain Ss in one of the goal arms or to force $\mathrm{S}$ to enter one arm on certain forced-choice trials. Interchangeable goal box walls made it possible to change the stimulus patterns between trials. The walls and floor of the maze, excluding the goal box walls, were painted flat gray, while the various interchangeable goal box walls were lined with one of five stimulus cards made from black tape and white cardboard: (1) black, (2) white, (3) 2 in. wide black and white horizontal stripes, (4) 1 in. black and white vertical stripes and (5) 1 in. black and white checkerboard squares. All Ss were placed on appropriate deprivation schedules two weeks prior to the experimental trials and handled in groups of four for $10 \mathrm{~min}$ a day. There were three levels of deprivation for each of the three experimental conditions. Deprivation schedules used were $0 \mathrm{~h}, 4 \mathrm{~h}$, and $22 \mathrm{~h}$. The three experimental conditions are as follows:

(a) Visual, direction, and odor cues available. The Ss were given a free-choice trial with both goal boxes having the white stimulus cards lining the walls. On Trial 2 Ss were forced to the same side they had entered on Trial 1 by lowering the retracing door for the nonentered arm. Both trials were rewarded. Between Trial 2 and 3 the walls of the nonentered goal box were changed to black, and on Trial $3 \mathrm{~S}$ was allowed a free-choice. Trials 1 and 2 serve to adapt $S$ to visual, direction, and odor cues associated with the entered arm. Since Ss can visually sample both alternatives on Trial 1 without making an entry, it is necessary to change the visual aspect of the stimulus in the nonentered arm between Trials 2 and 3. Assuming that spontaneous alternation is governed by Ss avoiding the repetition of a response to the cue or cues just previously encountered, all cues provide a basis for alternation on Trial 3.

(b) Direction and odor cues available. Trials 1 and 2 were the same as in the preceding group; however, no change was made in the nonentered goal box. Again Ss were given a free-choice trial on Trial 3 with no food available. The failure to change the visual cue of either arm between Trials 2 and 3 should reduce or eliminate any differential visual cue that might provide a basis for alternation.

(c) Visual cue available. Trial 1 was the same as the preceding two groups. Between Trials 1 and 2 the retracing door to the entered arm was lowered. The $\mathrm{S}$ was forced to enter the previously nonentered arm. Between Trials 2 and 3 the goal box walls of the nonentered arm (Trial 1) were changed to black for half of Ss and the goal box walls of the entered arms (Trial 1) were changed for the remaining Ss. On Trial $3 \mathrm{~S}$ was allowed a freechoice. Since Ss had equal experience with both arms, 
direction and odor are equated and only visual change could provide a basis for alternation on Trial 3.

The procedure was the same on the three subsequent days except the visual cues were different. On Trials 1 and 2 of Day 2, black walls were used, while the horizontal striped walls were used for the change condition. On Day 3, the horizontal striped walls were used on Trials 1 and 2 while the vertical striped walls were used for the change conditions. On Day 4, the vertical striped walls were used on Trials 1 and 2 while the checkerboard patterned walls were used in the change condition.

Assuming that Ss may adapt to monotonous conditions of stimulus change, increasingly complex stimuli were introduced in the stimulus change condition on subsequent days. The most complex stimulus card experienced on the previous day was used in both arms on Trials 1 and 2 of the following days. This procedure insured that Ss could not associate the cue value of any stimulus (e.g., horizontal stripes) with the change value (e.g., difference between a white stimulus and a horizontal stimulus on successive trials).

The Ss were run over three successive weeks, one drive level group per week. On the two days prior to the experiment each $\mathrm{S}$ was given $5 \mathrm{~min}$ adaptation in the stem of the maze with retracing doors present and gray goal boxes. On the four following days, each $S$ was given the appropriate experimental treatment. The three daily trials were given in immediate succession. The Ss were delayed in the start box for $15 \mathrm{sec}$ and again for $15 \mathrm{sec}$ in the entered maze arm or longer if Ss were still eating the four Noyes pellets provided for food reward. Results

There were a total of 360 test trials; 40 for each drive-cue condition. Each test trial represents a choice following two rewarded trials. The data recorded and analyzed were the proportion of alternation-nonalternation responses transformed by arc sine transformation. The failure to find a main effect or an interaction effect associated with days indicate that food reward was not effective in modifying amount of alternation. The significant interaction of drive and cue condition $(F=10.49$, $\mathrm{df}=4 / 12, \mathrm{p}<.005)$, but the nonsignificant effect due to drive level alone $(F=.89, d f=2 / 12, p>.05)$ indicate drive level did affect amount of alternation depending on the cues available to $S$. The data may be summarized as follows.

(a) When all the cues are available, alternation is independent of drive level $(85 \%, 73 \%$, and $78 \%$ alternation for low, moderate, and high drive levels). When certain cues are removed or neutralized, as in the following stimulus conditions, amount of alternation is related to drive level.

(b) Direction and/or odor cues appear to govern alternation most strongly under a moderate drive level ( $85 \%$ alternation); however, this effect is markedly reduced under low and high drive $(60 \%$ alternation for both low and high drive levels).

(c) Visual cues alone are most effective under high drive $(70 \%$ alternation, or in this condition, response to visual change). Apparently visual cues are utilized less under low and moderate drive levels when direction and odor have been neutralized (53\% alternation for both low and moderate drive levels).

\section{Discussion}

The results have an important bearing on the problem of cue utilization in spontaneous alternation. Our results provide evidence that drive levels affect which cues are utilized; however, the exact nature of this relation is not clear. The statement of Douglas (1966) that stimulus change is relatively unimportant in accounting for spontaneous alternation is not substantiated using the present design. Visual cues appear to be important provided that Ss are run under appropriate drive levels; provisions are made whereby visual sampling of the nonentered alternative is controlled and direction and odor cues are neutralized.

Relatively little work has been done on cue utilization as a function of drive levels. Typically, drive levels are assumed to influence incentive value of food or amount of drive reduction. Bruner, Matter, \& Papanek (1955) argue that drive level influences cue utilization; that peripheral or sensory stimulus may or may not be utilized in a task, such as a discrimination task, depending on the operation of internal processes such as drive levels produced by food deprivation. The data are consistent with this interpretation. While we have not demonstrated whether drive level affects number of cues utilized or specific cues utilized, the data tend to support the specific hypothesis. To the degree visual, direction, and odor cues affect exploration of the environment, drive levels may affect what is explored and, in all probability, what is learned as the result of exploration.

\section{References}

Baker, J. G., \& Franken, R. E. Alternation as a function of drive level and visual complexity. Psychon. Sci., 1967, 8.

Bruner, J. S., Matter, J., \& Papanek, M. L. Breadth of learning as a function of drive level and mechanization. Psychol. Rev., 1955, $62,1-10$.

Douglas, R. J. Cues for spontaneous alternation. J. comp. physiol. Psychol., 1966, 62, 171-183. 